\title{
On multiplicative metric spaces: survey
}

\author{
Tatjana Došenović ${ }^{1}$, Mihai Postolache ${ }^{2}$ and Stojan Radenović $3,4^{*}$
}

"Correspondence:

stojan.radenovic@tdt.edu.vn

${ }^{3}$ Nonlinear Analysis Research Group,

Ton Duc Thang University, Ho Chi

Minh City, Vietnam

${ }^{4}$ Department of Mathematics and

Statistics, Ton Duc Thang University,

Ho Chi Minh City, Vietnam

Full list of author information is

available at the end of the article

\begin{abstract}
The purpose of this survey is to prove that the fixed point results for various multiplicative contractions are in fact equivalent to the corresponding fixed point results in (standard) metric spaces. For example, such are recent results established by He et al. (Fixed Point Theory Appl. 2014:48, 2014), Mongkolkeha and Sintunavarat (J. Nonlinear Sci. Appl. 8:1134-1140, 2015) and Abdou (J. Nonlinear Sci. Appl. 9:2347-2363, 2016) and all others from the list of references. Our results here generalize, complement, and improve recent ones from existing literature.
\end{abstract}

MSC: Primary 47H10; secondary 54H25

Keywords: multiplicative metric space; fixed point; continuous; nondecreasing; lower semi-continuous mapping

\section{Introduction and preliminaries}

In 2008, Bashirov et al., among other things, initiated a new kind of spaces, called multiplicative metric spaces (MMS for short). The main idea was that the usual triangular inequality was replaced by a 'multiplicative triangle inequality' as follows:

Definition 1.1 ([4]) Let $X$ be a nonempty set. An operator $d^{*}: X \times X \rightarrow \mathbb{R}$ is a multiplicative metric (MM for short) on $X$, if it satisfies:

$\left(\mathrm{m} 1^{*}\right) d^{*}(x, y) \geq 1$ for all $x, y \in X$ and $d^{*}(x, y)=1$ if and only if $x=y$,

$\left(\mathrm{m} 2^{*}\right) d^{*}(x, y)=d^{*}(y, x)$ for all $x, y \in X$,

$\left(\mathrm{m} 3^{*}\right) d^{*}(x, z) \leq d^{*}(x, y) \cdot d^{*}(y, z)$ for all $x, y, z \in X$ (multiplicative triangle inequality).

If the operator $d^{*}$ satisfies $\left(\mathrm{m} 1^{*}\right)-\left(\mathrm{m} 3^{*}\right)$ then the pair $\left(X, d^{*}\right)$ is called a multiplicative metric space (MMS).

For more details of this new kind of spaces the reader can refer to [1-3, 5-16]. For example, it follows from these papers that an MMS $\left(X, d^{*}\right)$ is complete (sequentially compact) if and only if (standard) metric space (S-MS for short) $\left(X, \ln d^{*}\right)$ is such. Also, MM $d^{*}$ and (standard) metric (SM for short) ln $d^{*}$ induce the same topology on $X$ (see Theorem 2.1 below).

The next definition for S-MS is well known.

Definition 1.2 Let $X$ be a nonempty set. An operator $d: X \times X \rightarrow \mathbb{R}$ is a metric on $X$, if it satisfies:

(c) 2016 Došenović et al. This article is distributed under the terms of the Creative Commons Attribution 4.0 International License (http://creativecommons.org/licenses/by/4.0/), which permits unrestricted use, distribution, and reproduction in any medium, provided you give appropriate credit to the original author(s) and the source, provide a link to the Creative Commons license, and indicate if changes were made. 
(1) $d(x, y) \geq 0$ for all $x, y \in X$ and $d(x, y)=0$ if and only if $x=y$,

(2) $d(x, y)=d(y, x)$ for all $x, y \in X$,

(3) $d(x, z) \leq d(x, y)+d(y, z)$ for all $x, y, z \in X$ (standard triangle inequality).

If the operator $d$ satisfies (1)-(3) then the pair $(X, d)$ is called an S-MS.

Remark 1.3 It is clear that $d^{*}: X \times X \rightarrow[1,+\infty)$ while $d: X \times X \rightarrow[0,+\infty)$.

Very recently, the authors in [1] proved the following.

Theorem 1.4 ([1], Theorem 3.1) Let $S, T, A$, and $B$ be self-mappings of a complete $M M S$ $X$ satisfying the following conditions:

(i) $S X \subset B X, T X \subset A X$;

(ii) $A$ and $S$ are weakly commutative, $B$ and $T$ are also weakly commutative;

(iii) one of $S, T, A$ and $B$ is continuous;

(iv) $d^{*}(S x, T y) \leq\left(\max \left\{d^{*}(A x, B y), d^{*}(A x, S x), d^{*}(B y, T y), d^{*}(S x, B y), d^{*}(A x, T y)\right\}\right)^{\lambda}$, for some $\lambda \in\left(0, \frac{1}{2}\right)$, and all $x, y \in X$.

Then $S, T, A$, and B have a unique common fixed point (CFP for short).

Theorem 1.5 ([1], Theorem 3.2) Let S, T, $A$ and B be self-mappings of a complete $M M S$ $X$ satisfying the following conditions:

(i) $S X \subset B X, T X \subset A X$;

(ii) $A$ and $S$ are weakly commutative, $B$ and $T$ are also weakly commutative;

(iii) one of $S, T, A$ and $B$ is continuous;

(iv) $d^{*}\left(S^{p} x, T^{q} y\right) \leq\left(\max \left\{d^{*}(A x, B y), d^{*}\left(A x, S^{p} x\right), d^{*}\left(B y, T^{q} y\right), d^{*}\left(S^{p} x, B y\right)\right.\right.$, $\left.\left.d^{*}\left(A x, T^{q} y\right)\right\}\right)^{\lambda}$, for some $\lambda \in\left(0, \frac{1}{2}\right)$ and $p, q \in \mathbb{Z}^{+}$, and for all $x, y \in X$.

Then $S, T, A$, and $B$ have a unique CFP.

Remark 1.6 Since $\ln (\max \{a, b\})=\max \{\ln a, \ln b\}$ for all $a, b>0$ as well as $e^{\max \{a, b\}}=$ $\max \left\{e^{a}, e^{b}\right\}$ for all $a, b \in \mathbb{R}$, we see that the contractive condition (iv) of Theorem 1.4 and the following condition:

$$
\begin{aligned}
\ln d^{*}(S x, T y) \leq & \lambda \max \left\{\ln d^{*}(A x, B y), \ln d^{*}(A x, S x), \ln d^{*}(B y, T y),\right. \\
& \left.\ln d^{*}(S x, B y), \ln d^{*}(A x, T y)\right\},
\end{aligned}
$$

are equivalent; similarly, the contractive condition (iv) of Theorem 1.5 and the following condition:

$$
\begin{aligned}
\ln d^{*}\left(S^{p} x, T^{q} y\right) \leq & \lambda \max \left\{\ln d^{*}(A x, B y), \ln d^{*}\left(A x, S^{p} x\right), \ln d^{*}\left(B y, T^{q} y\right),\right. \\
& \left.\ln d^{*}\left(S^{p} x, B y\right), \ln d^{*}\left(A x, T^{q} y\right)\right\},
\end{aligned}
$$

are equivalent. This shows that both main results from [1] are equivalent with the corresponding ones in the framework of (S-MS).

Also recently Abdou [3] proved the following results. 
Theorem 1.7 ([3], Theorem 3.1) Let $\left(X, d^{*}\right)$ be a complete MMS. Let $S, T, A, B: X \rightarrow X$ be such that $S(X) \subset B(X), T(X) \subset A(X)$ and there exists $\lambda \in\left(0, \frac{1}{2}\right)$ such that

$$
\begin{aligned}
d^{* p}(S x, T y) \leq & {\left[\varphi \left(\operatorname { m a x } \left\{d^{* p}(A x, B y), \frac{d^{* p}(A x, S x) d^{* p}(B y, T y)}{1+d^{* p}(A x, B y)},\right.\right.\right.} \\
& \left.\left.\left.\frac{d^{* p}(A x, T y) d^{* p}(B y, A x)}{1+d^{* p}(A x, B y)}\right\}\right)\right]^{\lambda}
\end{aligned}
$$

for some $p \geq 1$ and all $x, y \in X$, where $\varphi:[0, \infty) \rightarrow[0, \infty)$ is an increasing function such that $\varphi(0)=0$ and $\varphi(t)<t$ for all $t>0$.

Suppose that one of the following conditions is satisfied:

(a) either $A$ or $S$ is continuous, the pair $(S, A)$ is compatible and the pair $(T, B)$ is weakly compatible;

(b) either $B$ or $T$ is continuous, the pair $(T, B)$ is compatible and the pair $(S, A)$ is weakly compatible.

Then $S, T, A$, and B have a unique CFP in X.

Theorem 1.8 ([3], Theorem 3.3) Let $\left(X, d^{*}\right)$ be a complete MMS. Let $S, T, A, B: X \rightarrow X$ be mappings such that $S(X) \subset B(X), T(X) \subset A(X)$ and there exists $\lambda \in\left(0, \frac{1}{2}\right)$ such that

$$
\begin{aligned}
d^{* p}\left(S^{m} x, T^{q} y\right) \leq & {\left[\varphi \left(\operatorname { m a x } \left\{d^{* p}(A x, B y), \frac{d^{* p}\left(A x, S^{m} x\right) d^{* p}\left(B y, T^{q} y\right)}{1+d^{* p}(A x, B y)},\right.\right.\right.} \\
& \left.\left.\left.\frac{d^{* p}\left(A x, T^{q} y\right) d^{* p}(B y, A x)}{1+d^{* p}(A x, B y)}\right\}\right)\right]^{\lambda}
\end{aligned}
$$

for some $p>1, m, q \in \mathbb{Z}^{+}$and for all $x, y \in X$, where $\varphi:[0, \infty) \rightarrow[0, \infty)$ is an increasing function such that $\varphi(0)=0$ and $\varphi(t)<t$ for all $t>0$.

Suppose that one of the following conditions is satisfied:

(a) either $A$ or $S$ is continuous, the pair $(S, A)$ is compatible and the pair $(T, B)$ is weakly compatible;

(b) either $B$ or $T$ is continuous, the pair $(T, B)$ is compatible and the pair $(S, A)$ is weakly compatible.

Then $S, T, A$, and $B$ have a unique CFP in $X$.

Remark 1.9 The first two of the following things are obvious in both previous theorems:

$1^{\circ} d^{* p}$ is also an MM on $X$.

$2^{\circ}$ It is sufficient that $\varphi:[1, \infty) \rightarrow[1, \infty)$ is an increasing function such that $\varphi(1)=1$ and $\varphi(t)<t$ for all $t>1$, further, for such $\varphi$ we get $\ln \varphi(t)<\ln t$ whenever $t>1$.

$3^{\circ}$ Also, it is clear enough that the function $\varphi$ in both previous theorems is superfluous. By removing it, (1) and (2) are, respectively, equivalent with

$$
\begin{aligned}
& \ln d^{* p}(S x, T y) \\
& \leq \lambda \max \left\{\ln d^{* p}(A x, B y), \ln d^{* p}(A x, S x)+\ln d^{* p}(B y, T y)-\ln \left(1+d^{* p}(A x, B y)\right),\right. \\
&\left.\ln d^{* p}(A x, T y)+\ln d^{* p}(B y, A x)-\ln \left(1+d^{* p}(A x, B y)\right)\right\}
\end{aligned}
$$


and

$$
\begin{aligned}
\ln d^{* p} & \left(S^{m} x, T^{q} y\right) \\
\leq & \lambda \max \left\{\ln d^{* p}(A x, B y), \ln d^{* p}\left(A x, S^{m} x\right)+\ln d^{* p}\left(B y, T^{q} y\right)-\ln \left(1+d^{* p}(A x, B y)\right),\right. \\
& \left.\ln d^{* p}\left(A x, T^{q} y\right)+\ln d^{* p}(B y, A x)-\ln \left(1+d^{* p}(A x, B y)\right)\right\} .
\end{aligned}
$$

Since $\left(X, \ln d^{* p}\right)$ is an S-MS, it follows that (3) and (4) are contractive conditions in the framework of S-MS.

\section{Main results}

Our first result generalizes Theorem 2.1 from [8] as well as Proposition 2.1 from [14]. The proof is immediate, i.e., by using the properties of functions $t \mapsto \ln t, t>0$ and $t \mapsto e^{t}$, therefore it is omitted.

Theorem 2.1 Let $\left(X, d^{*}\right)$ be an MMS. Then the pair $(X, d)$ is an S-MS where $d(x, y)=$ $\ln d^{*}(x, y)$ for all $x, y \in X$. Conversely, if $(X, d)$ is an $S$-MS then $\left(X, d^{*}\right)$ is an MMS where $d^{*}(x, y)=e^{d(x, y)}$ for all $x, y \in X$.

Hence, $\left(X, d^{*}\right)$ is an MMS if and only if $\left(X, \ln d^{*}\right)$ is an S-MS that is, $(X, d)$ is an S-MS if and only if $\left(X, e^{d}\right)$ is an MMS.

The Banach contraction principle [17], as one of the most important result in the fixed point theory and nonlinear analysis generally, has been generalized a lot, in metric as well as in different spaces which are generalizations of S-MS. Our motive in this section is to prove that various famous results, such as the Edelstein-Nemitskii, Boyd-Wong, and MeirKeeler ones, as well as some other well-known results, are equivalent in S-MS and MMS. In order to make the text easier to follow, in each of the cases, we will first expose a classical theorem in S-MS, after that we will give the appropriate theorem in MMS. Finally, we will prove the result which shows the equivalence between the two of them. The results will be exposed in historical order.

In 1962, Edelstein attempted to prove a fixed point theorem by keeping the completeness of the S-MS and replacing the Banach contractive condition by a slightly modified condition. It turned out that completeness is not a sufficient condition for the existence of fixed point for the new contractive condition. In $[12,18]$ a fixed point theorem for the proposed contraction conditions was proved, with certain restriction on the space.

Now we announce the well-known Edelstein-Nemytskii theorem in the framework of S-MS.

Theorem 2.2 ([12]) Let $(X, d)$ be a sequentially compact $S$-MS and let $f: X \rightarrow X$ satisfy the following condition:

$$
d(f x, f y)<d(x, y)
$$

whenever $x, y \in X$ and $x \neq y$. Then $f$ has a unique FP.

The appropriate fixed point theorem in the setting of MMS is the following one. 
Theorem 2.3 Let $\left(X, d^{*}\right)$ be a sequentially compact $M M S$ and let $f: X \rightarrow X$ satisfy the following condition:

$$
d^{*}(f x, f y)<d^{*}(x, y)
$$

whenever $x, y \in X$ and $x \neq y$. Then $f$ has a unique FP.

Our contribution in this research is the following result.

Theorem 2.4 Theorem 2.2 and Theorem 2.3 are equivalent.

Proof Let (5) hold. Then

$$
e^{d(f x, f y)}<e^{d(x, y)}
$$

So, we have $d^{*}(f x, f y)<d^{*}(x, y)$. Therefore (6) holds.

Suppose now that (6) holds. Then we have

$$
\ln d^{*}(f x, f y)<\ln d^{*}(x, y),
$$

i.e., $d(f x, f y)<d(x, y)$, so (5) is true.

The following well-known generalization of the Banach contraction principle was presented in 1969 in the paper of Boyd and Wong [19].

Theorem 2.5 ([19]) Let $(X, d)$ be a complete S-MS and let $f: X \rightarrow X$ satisfy, for all $x, y \in X$,

$$
d(f x, f y) \leq \psi_{1}(d(x, y))
$$

where $\psi_{1}:[0, \infty) \rightarrow[0, \infty)$ is upper semi-continuous from the right, satisfying $\psi_{1}(t)<t$ for $t>0$. Then $f$ has a unique FP, say $u \in X$, and $f^{n} x \rightarrow u$ as $n \rightarrow \infty$ for each $x \in X$.

Remark 2.6 It is easy to see that if $\psi_{1}(t)=\alpha(t) t, \alpha$ is increasing function such that $\alpha(t)<1$ for $t \geq 0$, then the Banach result follows.

The analogous result in MMS is presented below.

Theorem 2.7 Let $\left(X, d^{*}\right)$ be a complete $M M S$ and let $f: X \rightarrow X$ satisfy, for all $x, y \in X$,

$$
d^{*}(f x, f y) \leq \psi_{2}\left(d^{*}(x, y)\right)
$$

where $\psi_{2}:[1, \infty) \rightarrow[1, \infty)$ is upper semi-continuous from the right, satisfying $\psi_{2}(t)<t$ for $t>1$. Then $f$ has a unique $F P$, say $u \in X$, and $f^{n} x \rightarrow u$ as $n \rightarrow \infty$ for each $x \in X$.

The next theorem presents the equivalence between these two results.

Theorem 2.8 Theorem 2.5 and Theorem 2.7 are equivalent. 
Proof First of all, let (7) hold. It follows that

$$
e^{d(f x, f y)} \leq e^{\psi_{1}(d(x, y))}=e^{\psi_{1}\left(\ln d^{*}(x, y)\right)},
$$

that is,

$$
d^{*}(f x, f y) \leq e^{\psi_{1}\left(\ln d^{*}(x, y)\right)}=\psi_{2}\left(d^{*}(x, y)\right)
$$

where $\psi_{2}=\exp \circ \psi_{1} \circ \ln$. Hence, we see that (7) implies (8). Further, obviously $\psi_{2}:[1, \infty) \rightarrow$ $[1, \infty)$, together with $\psi_{1}$, is upper semi-continuous from the right, satisfying $\psi_{2}(t)<t$ for $t>1$.

Conversely, let (8) hold. From this we get

$$
\ln d^{*}(f x, f y) \leq \ln \left(\psi_{2}\left(d^{*}(x, y)\right)\right)=\ln \left(\psi_{2}\left(e^{d(x, y)}\right)\right)
$$

that is, we have

$$
d(f x, f y) \leq \ln \left(\psi_{2}\left(e^{d(x, y)}\right)\right)
$$

where $(X, d)$ is a complete S-MS. Taking in (9) $\psi_{1}=\ln \circ \psi_{2} \circ \exp$ we obtain (7), i.e.,

$$
d(f x, f y) \leq \psi_{1}(d(x, y)) .
$$

It is obvious that $\psi_{1}:[0, \infty) \rightarrow[0, \infty)$, together with $\psi_{2}$, is upper semi-continuous from the right, satisfying $\psi_{1}(t)<t$ for $t>0$. The proof of Theorem 2.5 is complete.

A very interesting theorem was proved in 1969 in the paper of Meir and Keeler [20]. They proved that the conclusion of the Banach contraction holds for a wider class of contraction mappings. Their contribution is the following theorem.

Theorem 2.9 ([20]) Let $(X, d)$ be a complete $S-M S$ and let $f: X \rightarrow X$ satisfy the following condition:

for each $\varepsilon>0$ there exists $\delta>0$ such that

$$
\varepsilon \leq d(x, y)<\varepsilon+\delta \Rightarrow d(f x, f y)<\varepsilon .
$$

Then $f$ has a unique FP, say $u \in X$ and for each $x \in X, \lim _{n \rightarrow \infty} f^{n} x=u$.

The respective theorem in MMS is the next one.

Theorem 2.10 Let $\left(X, d^{*}\right)$ be a complete $M M S$ and let $f$ be a self-mapping on $X$ satisfying the following condition:

for each $\varepsilon>1$ there exists $\delta>1$ such that

$$
\varepsilon \leq d^{*}(x, y)<\varepsilon \cdot \delta \Rightarrow d^{*}(f x, f y)<\varepsilon .
$$

Then $f$ has a unique FP, say $u \in X$ and for each $x \in X, \lim _{n \rightarrow \infty} f^{n} x=u$. 
As we have mentioned in this section, our contribution is to show that the above stated theorems are equivalent. So we prove the following theorem.

Theorem 2.11 Theorem 2.9 and Theorem 2.10 are equivalent.

Proof In fact we will prove that (11) implies (10) as well as, conversely, that (10) implies (11). Indeed, let (11) hold and let $\varepsilon>0$ be arbitrary. Then $e^{\varepsilon}>1$, so there exists $\delta>0$ (i.e., $\left.e^{\delta}>1\right)$ such that

$$
e^{\varepsilon} \leq e^{d(x, y)}=d^{*}(x, y)<e^{\varepsilon} \cdot e^{\delta} \Rightarrow d^{*}(f x, f y)=e^{d(f x, f y)}<e^{\varepsilon} .
$$

In other words (since ln and exp are strictly increasing functions),

$$
\varepsilon \leq d(x, y)<\varepsilon+\delta \Rightarrow d(f x, f y)<\varepsilon .
$$

Hence, (10) holds.

Conversely, let now (10) hold and let $\varepsilon>1$ be arbitrary. Then $\ln \varepsilon>0$, so there exists $\delta>1$ (i.e., $\ln \delta>0$ ) such that

$$
\ln \varepsilon \leq d(x, y)=\ln d^{*}(x, y)<\ln \varepsilon+\ln \delta \quad \Rightarrow \quad d(f x, f y)=\ln d^{*}(f x, f y)<\ln \varepsilon .
$$

In other words,

$$
\varepsilon \leq d^{*}(x, y)<\varepsilon \cdot \delta \quad \Rightarrow \quad d^{*}(f x, f y)<\varepsilon .
$$

Hence, (11) holds.

The proof is complete.

In the sequel, we consider the famous Kannan, Chatterje, and Zamfirescu results in both contexts. The Banach contractive condition implies the continuity of mapping $f$. Naturally, the question arises whether there is a contractive condition (sufficient to guarantee the existence of a fixed point) that does not imply continuity of the mapping $f$. The answer to the given question was published in 1968 in the Kannan paper [21], where he announced the following fixed point theorem.

Theorem $2.12([21,22])$ Let $(X, d)$ be a complete $S-M S, 0 \leq q<\frac{1}{2}$ and $f: X \rightarrow X$ be such that

$$
d(f x, f y) \leq q[d(x, f x)+d(y, f y)]
$$

for all $x, y \in X$. Then $f$ has a unique FP.

The analogous result in the framework of MMS is the following one.

Theorem 2.13 Let $\left(X, d^{*}\right)$ be a complete $M M S, 0 \leq q<\frac{1}{2}$ and $f: X \rightarrow X$ be such that

$$
d^{*}(f x, f y) \leq\left[d^{*}(x, f x) \cdot d^{*}(y, f y)\right]^{q},
$$


Now, our task is to show the equivalence between these two results. So we prove the following.

Theorem 2.14 Theorem 2.13 and Theorem 2.12 are equivalent.

Proof Let (13) hold. Then

$$
\begin{aligned}
d(f x, f y) & =\ln d^{*}(f x, f y) \\
& \leq q\left[\ln d^{*}(x, f x)+\ln d^{*}(y, f y)\right] \\
& =q[d(x, f x)+d(y, f y)],
\end{aligned}
$$

so (12) holds.

Let now (12) be satisfied. Then

$$
\begin{aligned}
d^{*}(f x, f y) & =e^{d(f x, f y)}=\left(e^{d(x, f x)}\right)^{q} \cdot\left(e^{d(y, f y)}\right)^{q} \\
& =\left(d^{*}(x, f x)\right)^{q} \cdot\left(d^{*}(y, f y)\right)^{q} \\
& =\left[d^{*}(x, f x) \cdot d^{*}(y, f y)\right]^{q} .
\end{aligned}
$$

Hence, we have proved Theorem 2.14.

A similar type of contractive condition was studied in 1972 by Chatterjea [23]. He proved the following result.

Theorem 2.15 Let $(X, d)$ be a complete $S-M S, 0 \leq q<\frac{1}{2}$ and $f: X \rightarrow X$ be such that

$$
d(f x, f y) \leq q[d(x, f y)+d(y, f x)]
$$

for all $x, y \in X$. Then $f$ has a unique FP.

The adequate theorem in terms of MMS is presented below.

Theorem 2.16 Let $\left(X, d^{*}\right)$ be a complete $M M S, 0 \leq q<\frac{1}{2}$ and $f: X \rightarrow X$ be such that

$$
d^{*}(f x, f y) \leq\left[d^{*}(x, f y) \cdot d^{*}(y, f x)\right]^{q},
$$

for all $x, y \in X$. Then $f$ has a unique FP.

Our contribution is the following theorem.

Theorem 2.17 Theorem 2.15 and Theorem 2.16 are equivalent.

Proof The proof follows using the same idea as in the previous one, so we omit it.

In 1972, Zamfirescu [24] united the theorems of Banach, Kannan, and Chatterjea. We give this theorem in both frameworks. 
Theorem 2.18 Let $(X, d)$ be a complete $S-M S, a_{i} \in[0,1)$ with $0 \leq a_{1}<1,0 \leq a_{2}, a_{3}<\frac{1}{2}$, and $f: X \rightarrow X$ be such that, for all $x, y \in X$, at least one of the following is true:

(i) $d(f x, f y) \leq a_{1}(d(x, y))$,

(ii) $\quad d(f x, f y) \leq a_{2}(d(x, f x)+d(y, f y))$,

(iii) $\quad d(f x, f y) \leq a_{3}(d(x, f y)+d(f x, y))$.

Then $f$ has a unique $F P$, say $u$, and for all $x \in X$ the sequence $f^{n}(x) \stackrel{d}{\rightarrow} u$ as $n \rightarrow \infty$.

Theorem 2.19 Let $\left(X, d^{*}\right)$ be a complete $M M S, a_{i} \in[0,1)$ with $0 \leq a_{1}<1,0 \leq a_{2}, a_{3}<\frac{1}{2}$, and $f: X \rightarrow X$ be such that, for all $x, y \in X$, at least one of the following is true:

$\left(\mathrm{i}^{*}\right) \quad d^{*}(f x, f y) \leq\left(d^{*}(x, y)\right)^{a_{1}}$,

(ii*) $\quad d^{*}(f x, f y) \leq\left(d^{*}(x, f x) d^{*}(y, f y)\right)^{a_{2}}$,

(iii*) $\quad d^{*}(f x, f y) \leq\left(d^{*}(x, f y) d^{*}(f x, y)\right)^{a_{3}}$.

Then $f$ has a unique FP, say $u$, and for all $x \in X$ the sequence $f^{n}(x) \stackrel{d^{*}}{\rightarrow} u$ as $n \rightarrow \infty$.

Theorem 2.20 Theorem 2.18 and Theorem 2.19 are equivalent.

Reich proved in 1971 [25] the following theorem and thus unified the Banach and Kannan theorems.

Theorem $2.21([22,25])$ Let $(X, d)$ be a complete $S-M S, a_{i} \in[0,1), i=1,2,3$ with $\sum_{i=1}^{3} a_{i}<$ 1 and $f: X \rightarrow X$ be such that

$$
d(f x, f y) \leq a_{1} d(x, y)+a_{2} d(x, f x)+a_{3} d(y, f y)
$$

for all $x, y \in X$. Then $f$ has a unique FP, say $u$, and for all $x \in X$ the sequence $f^{n}(x) \stackrel{d}{\rightarrow} u$ as $n \rightarrow \infty$.

Remark 2.22 Notice that one obtains the Banach fixed point theorem for $a=b=0$, as well as the Kannan theorem for $a=b$ and $c=0$.

Now we give the adequate theorem in the framework of MMS.

Theorem 2.23 Let $\left(X, d^{*}\right)$ be a complete MMS, $a_{i} \in[0,1), i=1,2,3$ with $\sum_{i=1}^{3} a_{i}<1$ and $f: X \rightarrow X$ be such that

$$
d^{*}(f x, f y) \leq\left(d^{*}(x, y)\right)^{a_{1}}\left(d^{*}(x, f x)\right)^{a_{2}}\left(d^{*}(y, f y)\right)^{a_{3}},
$$

for all $x, y \in X$. Then $f$ has a unique FP, say $u$, and for all $x \in X$ the sequence $f^{n}(x) \stackrel{d^{*}}{\rightarrow} u$ as $n \rightarrow \infty$.

In the same context, we present the equivalence between these two theorems. 
Theorem 2.24 Theorem 2.21 and Theorem 2.23 are equivalent.

Proof Let (14) be satisfied. Then

$$
\begin{aligned}
d^{*}(f x, f y) & =e^{d(f x, f y)} \\
& \leq e^{a_{1} d(x, y)+a_{2} d(x, f x)+a_{3} d(y, f y)} \\
& =d^{*}(x, y)^{a_{1}} d^{*}(x, f x)^{a_{2}} d^{*}(y, f y)^{a_{3}} d^{*}(x, f y) .
\end{aligned}
$$

Conversely, let (15) hold. Then

$$
\ln d^{*}(f x, f y) \leq a_{1} \ln d^{*}(x, y)+a_{2} \ln d^{*}(x, f x)+a_{3} \ln d^{*}(y, f y),
$$

so (14) holds.

In 1973, Hardy and Rogers [26] generalized Reich's [25] result and published the following interesting result. We present the mentioned theorem in both contexts.

Theorem 2.25 ([22]) Let $(X, d)$ be a complete S-MS, $a_{i} \in[0,1), i \in\{1,2,3,4,5\}$ with $\sum_{i=1}^{5} a_{i}<1$ and $f: X \rightarrow X$ be such that

$$
d(f x, f y) \leq a_{1} d(x, y)+a_{2} d(x, f x)+a_{3} d(y, f y)+a_{4} d(x, f y)+a_{5} d(f x, y),
$$

for all $x, y \in X$. Then $f$ has a unique $F P$, say $u$, and for all $x \in X$ the sequence $f^{n}(x) \stackrel{d}{\rightarrow} u$ as $n \rightarrow \infty$.

Theorem 2.26 Let $\left(X, d^{*}\right)$ be a complete $M M S, a_{i} \in[0,1), i \in\{1,2,3,4,5\}$ with $\sum_{i=1}^{5} a_{i}<1$ and $f: X \rightarrow X$ be such that

$$
d^{*}(f x, f y) \leq\left(d^{*}(x, y)\right)^{a_{1}}\left(d^{*}(x, f x)\right)^{a_{2}}\left(d^{*}(y, f y)\right)^{a_{3}}\left(d^{*}(x, f y)\right)^{a_{4}}\left(d^{*}(f x, y)\right)^{a_{5}},
$$

for all $x, y \in X$. Then $f$ has a unique $F P$, say $u$, and for all $x \in X$ the sequence $f^{n}(x) \stackrel{d^{*}}{\rightarrow} u$ as $n \rightarrow \infty$.

We notice that these two theorems are equivalent, i.e., the following holds.

Theorem 2.27 Theorem 2.25 and Theorem 2.26 are equivalent.

Proof Since the idea is the same in all theorems so far, to avoid repeating we give this theorem without proof.

In 1973, Geraghty [27] generalized the Banach contraction principle by considering an auxiliary function in the following way.

Theorem 2.28 ([27]) Let $(X, d)$ be a complete $S$-MS and let $f: X \rightarrow X$ satisfy the following condition: there exists $\beta_{1}:[0, \infty) \rightarrow[0,1)$ such that if $\beta_{1}\left(t_{n}\right) \rightarrow 1$ then $t_{n} \rightarrow 0$ and for all $x, y \in X$ the following holds:

$$
d(f x, f y) \leq \beta_{1}(d(x, y)) d(x, y) .
$$

Then $f$ has a unique FP, say $u \in X$, and for each $x \in X, \lim _{n \rightarrow \infty} f^{n} x=u$. 
In the sequel we consider a Geraghty type contraction in MMS. Consider the functions $\beta:[1, \infty) \rightarrow[0,1)$ such that if $\beta\left(t_{n}\right) \rightarrow 1$ then $t_{n} \rightarrow 1$. One such function $\beta$ is $\beta(t)=e^{1-t}$, $t \geq 1$. Now we formulate the Geraghty type theorem in the framework of MMS.

Theorem 2.29 Let $\left(X, d^{*}\right)$ be a complete $M M S$ and let $f: X \rightarrow X$ satisfy the following condition: there exists $\beta_{2}:[1, \infty) \rightarrow[0,1)$ such that if $\beta_{2}\left(t_{n}\right) \rightarrow 1$ then $t_{n} \rightarrow 1$ and for all $x, y \in X$ the following holds:

$$
d^{*}(f x, f y) \leq\left(d^{*}(x, y)\right)^{\beta_{2}\left(d^{*}(x, y)\right)} .
$$

Then $f$ has a unique FP, say $u \in X$, and for each $x \in X, \lim _{n \rightarrow \infty} f^{n} x=u$.

Also, for a Geraghty type contraction in both frameworks we have the following.

Theorem 2.30 Theorem 2.28 and Theorem 2.29 are equivalent.

Proof Let (16) hold. Then we have

$$
e^{d(f x, f y)} \leq e^{\beta_{1}(d(x, y)) d(x, y)}=\left(e^{d(x, y)}\right)^{\beta_{1}(d(x, y))},
$$

i.e.,

$$
d^{*}(f x, f y) \leq\left(d^{*}(x, y)\right)^{\beta_{1}\left(\ln d^{*}(x, y)\right)}=\left(d^{*}(x, y)\right)^{\beta_{2}\left(d^{*}(x, y)\right)},
$$

where $\beta_{2}=\beta_{1} \circ \ln$. Clearly, $\beta_{2}:[1, \infty) \rightarrow[0,1)$ with $\beta_{2}\left(t_{n}\right) \rightarrow 1 \Rightarrow t_{n} \rightarrow 1$. We have proved that (16) implies (17).

Conversely, it follows from (17) that

$$
\ln d^{*}(f x, f y) \leq \beta_{2}\left(d^{*}(x, y)\right) \ln d^{*}(x, y)
$$

or, equivalently,

$$
d(f x, f y) \leq \beta_{2}\left(e^{d(x, y)}\right) d(x, y)=\beta_{1}(d(x, y)) d(x, y)
$$

where $\beta_{1}=\beta_{2}$ o exp. It is obvious that $\beta_{1}:[0, \infty) \rightarrow[0,1)$ with $\beta_{1}\left(t_{n}\right) \rightarrow 1 \Rightarrow t_{n} \rightarrow 0$. This means that (17) implies (16), that is, Theorem 2.11 and Theorem 2.29 are equivalent.

Another generalization of the Kannan fixed point theorem was given by Bianchini [28] in 1972, i.e., the following very interesting result was proved.

Theorem 2.31 ([28]) Let $(X, d)$ be a complete $S-M S, \lambda \in[0,1)$ and $f: X \rightarrow X$ be such that

$$
d(f x, f y) \leq \lambda \max \{d(x, f x), d(y, f y)\}
$$

for all $x, y \in X$. Then $f$ has a unique FP, say $u$, and for all $x \in X$ the sequence $f^{n}(x) \stackrel{d}{\rightarrow} u$ as $n \rightarrow \infty$. 
The corresponding result in MMS is the following.

Theorem 2.32 Let $\left(X, d^{*}\right)$ be a complete $M M S, \lambda \in[0,1)$ and $f: X \rightarrow X$ be such that

$$
d^{*}(f x, f y) \leq\left(\max \left\{d^{*}(x, f x), d^{*}(y, f y)\right\}\right)^{\lambda},
$$

for all $x, y \in X$. Then $f$ has a unique $F P$, say $u$, and for all $x \in X$ the sequence $f^{n}(x) \stackrel{d}{\rightarrow} u$ as $n \rightarrow \infty$.

We have the next result.

Theorem 2.33 Theorem 2.31 and Theorem 2.32 are equivalent.

Proof Omitted.

One of the most general contraction conditions was given by Cirić [29] in 1974. He defined and investigated quasicontractions, mappings that unified the Banach, Kannan, Chatterjea, and Bianchini contractions. We announce this theorem in both frameworks.

Theorem $2.34([22,29])$ Let $(X, d)$ be a complete $S-M S, \lambda \in[0,1)$ and $f: X \rightarrow X$ be such that

$$
d(f x, f y) \leq \lambda \max \{d(x, y), d(x, f x), d(y, f y), d(x, f y), d(f x, y)\}
$$

for all $x, y \in X$. Then $f$ has a unique $F P$, say $u$, and for all $x \in X$ the sequence $f^{n}(x) \stackrel{d}{\rightarrow} u$ as $n \rightarrow \infty$.

The expected result within the MMS is presented below.

Theorem 2.35 Let $\left(X, d^{*}\right)$ be a complete $M M S, \lambda \in[0,1)$ and $f: X \rightarrow X$ be such that

$$
d^{*}(f x, f y) \leq\left(\max \left\{d^{*}(x, y), d^{*}(x, f x), d^{*}(y, f y), d^{*}(x, f y), d^{*}(f x, y)\right\}\right)^{\lambda},
$$

for all $x, y \in X$. Then $f$ has a unique FP, say $u$, and for all $x \in X$ the sequence $f^{n}(x) \stackrel{d^{*}}{\rightarrow} u$ as $n \rightarrow \infty$.

Theorem 2.36 Theorem 2.34 and Theorem 2.35 are equivalent.

Proof Omitted.

Sehgal in 1969 [30], proved an FP theorem using a condition that involves contractive iterate at each point of the space, where the space is complete and the mapping is continuous. Soon afterwards, Guseman in 1970 [31] generalized the Sehgal results for mappings which are not necessarily continuous.

Theorem $2.37([30,31])$ Let $(X, d)$ be a complete $S-M S$ and $: X \rightarrow X$ a mapping satisfying the condition: there exists $\lambda<1$ such that, for every $x \in X$, there exists $k(x) \in \mathbb{N}$ such that 
for all $y \in X$ the following is satisfied:

$$
d\left(f^{k(x)} x, f^{k(x)} y\right) \leq \lambda d(x, y)
$$

Then $f$ has a unique FP, say $u \in X$, and $f^{n} x \rightarrow u$ for each $x \in X$.

The corresponding result in the framework of MMS is the following.

Theorem 2.38 Let $\left(X, d^{*}\right)$ be a complete $M M S$ and $f: X \rightarrow X$ a mapping satisfying the condition: there exists $\lambda<1$ such that, for every $x \in X$, there exists $k(x) \in \mathbb{N}$ such that for all $y \in X$ the following is satisfied:

$$
d^{*}\left(f^{k(x)} x, f^{k(x)} y\right) \leq\left(d^{*}(x, y)\right)^{\lambda}
$$

Then $f$ has a unique FP, say $u \in X$, and $f^{n} x \rightarrow u$ for each $x \in X$.

Our expected result is the following.

Theorem 2.39 Theorem 2.37 and Theorem 2.38 are equivalent.

Proof Omitted.

In 2001, Rhoades [32] came up with the following idea: the Banach contraction can be interpreted as $d(f x, f y) \leq d(x, y)-\varphi(d(x, y))$. This extension is called the weakly contractive mapping, and in the same paper the following interesting result was proved.

Theorem 2.40 ([32]) Let $(X, d)$ be a complete $S$-MS and $f: X \rightarrow X$ a mapping satisfying the condition:

$$
d(f x, f y) \leq d(x, y)-\varphi_{1}(d(x, y))
$$

for all $x, y \in X$, where $\varphi_{1}:[0,+\infty) \rightarrow[0,+\infty), \varphi_{1}$ is lower semi-continuous and $\varphi_{1}^{-1}(\{0\})=$ $\{0\}$. Then $f$ has a unique $F P$, say $u \in X$, and $f^{n} x \rightarrow u$ for each $x \in X$.

The analogous result in the framework of MMS is the following.

Theorem 2.41 Let $\left(X, d^{*}\right)$ be a complete $M M S$ and $f: X \rightarrow X$ a mapping satisfying the condition:

$$
d^{*}(f x, f y) \leq \frac{d^{*}(x, y)}{\varphi_{2}\left(d^{*}(x, y)\right)}
$$

for all $x, y \in X$, where $\varphi_{2}:[1,+\infty) \rightarrow[1,+\infty), \varphi_{2}$ is lower semi-continuous and $\varphi_{2}^{-1}(\{1\})=$ $\{1\}$. Then $f$ has a unique $F P$, say $u \in X$, and $f^{n} x \rightarrow u$ for each $x \in X$.

Finally, we have the next result.

Theorem 2.42 Theorem 2.40 and Theorem 2.41 are equivalent. 
Proof Omitted.

Let $(X, d)$ be a metric space, $K \subseteq X$ and $\varphi: K \rightarrow[0,+\infty)$ a function on $K$. Suppose that $\varphi$ is a lower semi-continuous (l.s.c. for short) function on $K$, i.e.,

$$
\lim _{n \rightarrow \infty} x_{n}=x \text { implies } \varphi(x) \leq \underline{\lim \varphi\left(x_{n}\right)}
$$

and let $f: K \rightarrow K$ be an arbitrary self-mapping on $K$ such that

$$
d(x, f x) \leq \varphi(x)-\varphi(f x)
$$

If $K$ is a nonempty compact subset of $X$, then it is well known that $\varphi$ attains its minimum at some point of $K$, say $z$. So if $f: K \rightarrow K$, then $\varphi(f z) \geq \varphi(z)$ and, from (18),

$$
0 \leq d(z, f z) \leq \varphi(z)-\varphi(f z) \leq 0
$$

Hence $d(z, f z)=0$. Therefore, $f$ has a FP.

For MMS we have the analogous result:

Let $\left(X, d^{*}\right)$ be a MMS, $K \subseteq X$ and $\varphi^{*}: K \rightarrow[1,+\infty)$ a function on $K$. Suppose that $\varphi^{*}$ is an l.s.c. function on $K$, and let $f: K \rightarrow K$ be an arbitrary self-mapping on $K$ such that

$$
d^{*}(f x, f y) \leq \frac{\varphi^{*}(x)}{\varphi^{*}(f x)}
$$

for all $x, y \in X$.

The above result for compact metric spaces is generalized to complete metric spaces by Caristi-Kirk. It is well known that Caristi-Kirk's (or Caristi-Kirk-Browder's) theorem is essentially equivalent to the Ekeland variational principle [33]. The original proof of Caristi-Kirk's theorem was rather complicated and several new proofs were presented (see, e.g., [34]).

Here we give this famous result.

Theorem 2.43 (Caristi [35], 1976) Let $(X, d)$ be a complete metric space and $\phi: X \rightarrow$ $[0,+\infty)$ be an l.s.c.function. Then any mapping $f: X \rightarrow X$ satisfying (18) has an FP in $X$.

The corresponding result in the framework of MMS is the following.

Theorem 2.44 Let $\left(X, d^{*}\right)$ be a complete MMS and $f: X \rightarrow X$ a mapping satisfying the condition

$$
d^{*}(f x, f y) \leq \frac{\phi^{*}(x)}{\phi^{*}(f x)}
$$

for all $x, y \in X$, where $\phi^{*}:[1,+\infty) \rightarrow[1,+\infty)$, $\phi^{*}$ is an l.s.c. function. Then $f$ has an FP in $X$.

Now, we have the following result.

Theorem 2.45 Theorems 2.43 and 2.44 are equivalent. 
Proof Omitted.

In 1965, Prešić [36] extended the Banach contraction mapping principle to mappings defined on product spaces and proved the following theorem.

Theorem 2.46 Let $(X, d)$ be a complete metric space, $k$ a positive integer, and $f: X^{k} \rightarrow X$ a mapping satisfying the following contractive type condition:

$$
d\left(f\left(x_{1}, x_{2}, \ldots, x_{k}\right), f\left(x_{2}, x_{3}, \ldots, x_{k+1}\right)\right) \leq \sum_{i=1}^{k} q_{i} d\left(x_{i}, x_{i+1}\right),
$$

for every $x_{1}, x_{2}, \ldots, x_{k+1} \in X$, where $q_{1}, q_{2}, \ldots, q_{k}$ are nonnegative constants such that $q_{1}+q_{2}+$ $\cdots+q_{k}<1$. Then there exists a unique point $x \in X$ such that $f(x, x, \ldots, x)=x$. Moreover, if $x_{1}, x_{2}, \ldots, x_{k}$ are arbitrary points in $X$ and, for $n \in \mathbb{N}$,

$$
x_{n+k}=f\left(x_{n}, x_{n+1}, \ldots, x_{n+k}\right)
$$

then the sequence $\left\{x_{n}\right\}$ is convergent and

$$
\lim _{n \rightarrow \infty} x_{n}=f\left(\lim _{n \rightarrow \infty} x_{n}, \lim _{n \rightarrow \infty} x_{n}, \ldots, \lim _{n \rightarrow \infty} x_{n}\right)
$$

The analogous result in the framework of MMS is the following.

Theorem 2.47 Let $\left(X, d^{*}\right)$ be a complete metric space, $k$ a positive integer, and $f: X^{k} \rightarrow X$ a mapping satisfying the following contractive type condition:

$$
d^{*}\left(f\left(x_{1}, x_{2}, \ldots, x_{k}\right), f\left(x_{2}, x_{3}, \ldots, x_{k+1}\right)\right) \leq \prod_{i=1}^{k} d^{*}\left(x_{i}, x_{i+1}\right)^{q_{i}}
$$

for all $x_{1}, x_{2}, \ldots, x_{k+1} \in X$, where $q_{1}, q_{2}, \ldots, q_{k}$ are nonnegative constants such that $q_{1}+q_{2}+$ $\cdots+q_{k}<1$. Then there exists a unique point $x \in X$ such that $f(x, x, \ldots, x)=x$. Moreover, if $x_{1}, x_{2}, \ldots, x_{k}$ are arbitrary points in $X$ and, for $n \in \mathbb{N}$,

$$
x_{n+k}=f\left(x_{n}, x_{n+1}, \ldots, x_{n+k}\right)
$$

then the sequence $\left\{x_{n}\right\}$ is convergent and

$$
\lim _{n \rightarrow \infty} x_{n}=f\left(\lim _{n \rightarrow \infty} x_{n}, \lim _{n \rightarrow \infty} x_{n}, \ldots, \lim _{n \rightarrow \infty} x_{n}\right) .
$$

Finally, our result is the following.

Theorem 2.48 Theorem 2.46 and Theorem 2.47 are equivalent.

Proof Omitted.

Remark 2.49 According to [37], new results are possible. 


\section{Conclusion}

Every known contractive condition, that is, the FP result (theorem) in the framework of S-MS has an analog in the MMS and conversely. These analogs are equivalent. In particular, this is true for all results in $[1-7,11,13,16,38]$.

\section{Further work}

Encouraged by this research, the idea for the following definitions emerged.

Definition 4.1 Let $X \neq \emptyset$ and let $s \geq 1$ be a given real number. A function $d_{b}^{*}: X \times X \rightarrow \mathbb{R}^{+}$ is called a $b$-MM, provided that, for all $x, y, z \in X$,

(1) $d_{b}^{*}(x, y)=0$ iff $x=y$;

(2) $d_{b}^{*}(x, y)=d_{b}^{*}(y, x)$;

(3) $d_{b}^{*}(x, z) \leq\left[d_{b}^{*}(x, y) \cdot d_{b}^{*}(y, z)\right]^{s}$.

The pair $\left(X, d_{b}^{*}\right)$ is called a $b$-MMS.

Definition 4.2 A partial MM on a (nonempty) set $X$ is a function $p^{*}: X \times X \rightarrow \mathbb{R}^{+}$such that for all $x, y, z \in X$ the following are satisfied:

(1) $x=y \Leftrightarrow p^{*}(x, x)=p^{*}(x, y)=p^{*}(y, y)$;

(2) $p^{*}(x, x) \leq p^{*}(x, y)$;

(3) $p^{*}(x, y)=p^{*}(y, x)$;

(4) $p^{*}(x, z) \leq \frac{p^{*}(x, y) \cdot p^{*}(y, z)}{p^{*}(y, y)}$.

A partial MMS is a pair $\left(X, p^{*}\right)$ such that $X \neq \emptyset$ and $p^{*}$ is a partial MM on $X$.

For future work, it would be interesting to research analogous results with well-known results in $b$-metric and partial metric spaces, respectively.

\section{Competing interests}

The authors declare that they have no competing interests.

\section{Authors' contributions}

All authors contributed equally to the writing of this paper. All authors read and approved the final manuscript.

\section{Author details}

${ }^{1}$ Faculty of Technology, University of Novi Sad, Bulevar cara Lazara 1, Novi Sad, Serbia. ${ }^{2}$ Faculty of Applied Sciences, University Politehnicaiof Bucharest, Bucharest, 060042, Romania. ${ }^{3}$ Nonlinear Analysis Research Group, Ton Duc Thang University, Ho Chi Minh City, Vietnam. ${ }^{4}$ Department of Mathematics and Statistics, Ton Duc Thang University, Ho Chi Minh City, Vietnam.

\section{Acknowledgements}

The authors are indebted to anonymous referees who helped us to improve this text. The first author is thankful to Ministry of Education, Sciences and Technological Development of Serbia.

Received: 27 March 2016 Accepted: 16 September 2016 Published online: 27 September 2016

\section{References}

1. He, X, Song, M, Chen, D: Common fixed points for weak commutative mappings on a multiplicative metric space. Fixed Point Theory Appl. 2014, 48 (2014)

2. Mongkolkeha, C, Sintunavarat, W: Best proximity points for multiplicative proximal contraction mapping on multiplicative metric spaces. J. Nonlinear Sci. Appl. 8, 1134-1140 (2015)

3. Abdou, AAN: Fixed point theorems for generalized contraction mappings in multiplicative metric spaces. J. Nonlinear Sci. Appl. 9, 2347-2363 (2016)

4. Bashirov, A, Kurpinar, E, Ozyapici, A: Multiplicative calculus and its applications. J. Math. Anal. Appl. 337(1), 36-48 (2008)

5. Abbas, M, Ali, B, Suleiman, Y: Common fixed points of locally contractive mappings in multiplicative metric spaces with applications. Int. J. Math. Math. Sci. 2015, Article ID 218683 (2015)

6. Abbas, M, De La Sen, M, Nazir, T: Common fixed points of generalized rational type cocyclic mappings in multiplicative metric spaces. Discrete Dyn. Nat. Soc. 2015, Article ID 532725 (2015) 
7. Abodayeh, K, Pitea, A, Shatanawi, W, Abdeljawad, T: Remarks on multiplicative metric spaces and related fixed points (2015). arXiv:1512.03771v1 [math.GN]

8. Agarwal, RP, Karapinar, E, Samet, B: An essential remark on fixed point results on multiplicative metric spaces. Fixed Point Theory Appl. 2016, 21 (2016)

9. Došenović, T, Radenović, S: Some critical remarks on the paper: 'An essential remark on fixed point results on multiplicative metric spaces.' J. Adv. Math. Stud. (in press)

10. Grossman, M, Katz, R: Non-Newtonian Calculus. Lee Press, Pigeon Cove (1972)

11. Kang, SM, Kumar, P, Kumar, S, Nagpal, P, Garg, SK: Common fixed points for compatible mappings and its variants in multiplicative metric spaces. Int. J. Pure Appl. Math. 102(2), 383-406 (2015)

12. Nemytzki, W: The fixed point method in analysis. Usp. Mat. Nauk 1, 141-174 (1936) (in Russian)

13. Özavsar, M, Cevikel, AC: Fixed points of multiplicative contraction mappings on multiplicative metric spacers (2012). arXiv:1205.5131v1 [math.GM]

14. Shukla, S: Some critical remarks on the multiplicative metric spaces and fixed point results. J. Adv. Math. Stud. (2017, to appear)

15. Stanley, D: A multiplicative calculus. PRIMUS IX(4), 310-326 (1999)

16. Yamaod, $\mathrm{O}$, Sintunavarat, $\mathrm{W}$ : Some fixed point results for generalized contraction mappings with cyclic $(\alpha, \beta)$-admissible mapping in multiplicative metric spaces. J. Inequal. Appl. 2014, 488 (2014)

17. Banach, S: Sur les opérations dans les ensembles abstraits et leur application aux équations intégrales. Fundam. Math. 3, 133-181 (1922)

18. Edelstein, M: On fixed and periodic points under contractive mappings. J. Lond. Math. Soc. 37, 74-79 (1962)

19. Boyd, DW, Wong, JS: On linear contractions. Proc. Am. Math. Soc. 20, $458-464$ (1969)

20. Meir, A, Keeler, E: A theorem on contraction mappings. J. Math. Anal. Appl. 28, 326-329 (1969)

21. Kannan, R: Some results on fixed points. Bull. Calcutta Math. Soc. 60, 71-76 (1968)

22. Rhoades, BE: A comparison of various definitions of contractive mappings. Trans. Am. Math. Soc. 226, 257-290 (1977)

23. Chatterjea, SK: Fixed point theorems. C. R. Acad. Bulgare Sci. 25, 727-730 (1972)

24. Zamfirescu, T: Fixed point theorems in metric spaces. Arch. Math. 23, 292-298 (1972)

25. Reich, S: Some remarks concerning contraction mappings. Can. Math. Bull. 14, 121-124 (1971)

26. Hardy, GE, Rogers, TD: A generalization of a fixed point theorem of Reich. Can. Math. Bull. 16, 201-206 (1973)

27. Geraghty, M: On contractive mappings. Proc. Am. Math. Soc. 40, 604-608 (1973)

28. Bianchini, RMT: Su un problema di S. Reich riguardante la teoria dei punti fissi. Boll. UMI 5(4), 103-106 (1972)

29. Ćirić, LB: A generalization of Banach's contraction principle. Proc. Am. Math. Soc. 45, 267-273 (1974)

30. Sehgal, VM: A fixed point theorem for mappings with a contractive iterate. Proc. Am. Math. Soc. 23, 633-634 (1969)

31. Guseman, LF: Fixed point theorems for mappings with a contractive iterate at a point. Proc. Am. Math. Soc. 26 615-618 (1970)

32. Rhoades, BE: Some theorems on weakly contractive maps. Nonlinear Anal. 47, 2683-2693 (2001)

33. Ekeland, I: Sur les problemmes variationels. C. R. Acad. Sci. Paris 275, 1057-1059 (1972)

34. Pasicki, L: A short proof of the Caristi theorem. Comment. Math. Prace Mat. 20, 427-428 (1977/78)

35. Caristi, J: Fixed point theorems for mappings satisfying inwardness conditions. Trans. Am. Math. Soc. 215, 241-251 (1976)

36. Prešić, SB: Sur la convergence des suites. C. R. Acad. Sci. Paris 260, 3828-3830 (1965)

37. Ćirić, LB, Prešić, SB: On Prešić type generalization of the Banach contraction mapping principle. Acta Math. Univ. Comen. 76(2), 143-147 (2007)

38. Sarwar, M, Badshah-e-Rome: Some unique fixed point theorems in multiplicative metric space (2014). arXiv:1410.3384v2 [math.GM]

\section{Submit your manuscript to a SpringerOpen ${ }^{\ominus}$ journal and benefit from:}

- Convenient online submission

Rigorous peer review

- Immediate publication on acceptance

- Open access: articles freely available online

- High visibility within the field

- Retaining the copyright to your article 